\title{
Human parvovirus B19 nonstructural protein NS1 activates NLRP3 inflammasome signaling in adult-onset Still's disease
}

\author{
DER-YUAN CHEN ${ }^{1-4}$, YI-MING CHEN ${ }^{1-3^{*}}$, HSIN-HUA CHEN ${ }^{1-3^{*}}$, CHIA-WEI HSIEH $^{1-3}$, \\ NING-RONG GUNG ${ }^{1}$, WEI-TING HUNG ${ }^{1,2}$, BOR-SHOW TZANG ${ }^{4-7}$ and TSAI-CHING HSU ${ }^{4,5,7}$ \\ ${ }^{1}$ Department of Medical Education and Research, Taichung Veterans General Hospital, Taichung 40705; \\ ${ }^{2}$ Faculty of Medicine, National Yang Ming University, Taipei 11221; ${ }^{3}$ Institute of Biomedical Science and Rong Hsing \\ Research Center for Translational Medicine, National Chung Hsing University, Taichung 40227; ${ }^{4}$ Institute of Biochemistry, \\ Microbiology and Immunology; ${ }^{5}$ Immunology Research Center; ${ }^{6}$ Department of Biochemistry, School of Medicine; \\ ${ }^{7}$ Clinical Laboratory, Chung Shan Medical University, Taichung 40201, Taiwan, R.O.C.
}

Received January 18, 2017; Accepted November 13, 2017

DOI: $10.3892 / \mathrm{mmr} .2017 .8275$

\begin{abstract}
Dysregulation of inflammasomes serves a pathogenic role in autoinflammatory diseases (AIDs) and adult-onset Still's disease (AOSD) has been categorized as an AID. The present study investigated the expression of NLR family pyrin domain containing proteins (NLRPs) inflammasome in patients with AOSD, the effect of inflammasome inhibitors on NLRP3 signaling and whether human parvovirus B19-associated antigens can activate NLRP3 in patients with AOSD. mRNA expression levels of NLRPs in peripheral blood mononuclear cells (PBMCs) from 34 patients with AOSD and 14 healthy individuals were determined using reverse transcription-quantitative polymerase chain reaction. Protein expression of NLRP3 was evaluated by western blotting. Supernatant cytokine levels were measured by ELISA. Among the NLRPs investigated in the present study, NLRP3 transcripts were markedly elevated and expression of NLRP2, NLRP7 and NLRP12 was decreased in patients with AOSD compared with the controls. Treatment with NLRP3 inhibitors significantly reduced downstream NLRP3 signaling in PBMCs form patients with AOSD. B19-nonstructural protein (NS)1 stimulation of PBMCs from patients with AOSD
\end{abstract}

Correspondence to: Professor Bor-Show Tzang, Department of Biochemistry, School of Medicine, Chung Shan Medical University, 110 Sector 1, Jianguo N. Road, Taichung 40201, Taiwan, R.O.C.

E-mail: bstzang@csmu.edu.tw

Professor Tsai-Ching Hsu, Institute of Biochemistry, Microbiology and Immunology, Chung Shan Medical University, 110 Sector 1, Jianguo N. Road, Taichung 40201, Taiwan, R.O.C.

E-mail: htc@csmu.edu.tw

${ }^{*}$ Contributed equally

Key words: nucleotide-binding oligomerization, NLR family pyrin domain containing proteins, inflammasome, parvovirus B19 nonstructural protein NS1, adult-onset Still's disease induced significant upregulation of transcript levels of NLRP3, caspase- 1 and interleukin (IL)-1 $\beta$ compared with PBMCs from healthy controls. B19-NS1 stimulation of PBMCs from patients with AOSD induced significant increase in supernatant levels of IL-1 $\beta$ and protein expression of NLRP3, caspase-1, IL-1 $\beta$, and IL-18 compared with healthy controls. Elevated expression of NLRP3 and its downstream inflammasome signaling components in patients with AOSD indicated a potential pathogenic role of B19-NS1. Thus, B19-NS1 may induce expression of IL-1 $\beta$ and IL-18 through activation of caspase-1-associated NLRP3-inflammasome in AOSD.

\section{Introduction}

Nucleotide-binding domain leucine-rich repeat-containing receptors (NLRs) are the main constituents of inflammasomes and serve a role in innate immunity and inflammation (1-3). NLR family pyrin domain containing proteins (NLRPs) are the largest subfamily of NLRs $(4,5)$. Activation of NLRs results in recruitment of caspase-1 to inflammasomes via an adaptor protein that contains a pyrin domain (PYD) and caspase recruitment domain [CARD; PYD and CARD domain containing (PYCARD) protein] and caspase-1 subsequently cleaves pro-interleukin (IL)-1 $\beta$ and pro-IL-18 to produce their active forms (1-3).

Previous studies revealed that deregulated inflammasomes serve an important role in the pathogenesis of autoinflammatory diseases (AIDs) (6-8). AIDs are characterized by episodes of abnormally increased inflammation without production of high-titer autoantibodies or antigen-specific T lymphocytes, but with symptoms including spiking fever, erythema, arthropathy, serositis and vasculopathy (9-11). Adult-onset Still's disease (AOSD) is an inflammatory disorder characterized by spiking fever, rash, arthritis, hepatosplenomegaly, variable multisystemic involvement and increases in levels of acute phase reactants $(12,13)$. AOSD has been classified as an AID as it demonstrates similar phenotypes without significantly elevated levels of autoantibodies $(11,14)$. Previous studies have revealed increased levels of serum IL-1 $\beta$ and IL-18 in patients with AOSD (15-18). We further hypothesized that 
inflammasome-dependent processing of cytokines may have a role in in AOSD pathogenesis. However, to the best of our knowledge there is no data available on the pathogenic role of inflammasomes in AOSD.

Human parvovirus B19 (B19) is a DNA virus responsible for a wide range of human diseases (19). It consists of two structural proteins, VP1 and VP2, which are identical except for a 227-amino acids long amino-terminal end of VP1, known as the VP1-unique region (VP1u) (20). B19-VP1u is essential for cytotoxicity and infectivity, while B19 non-structural protein-1 (B19-NS1) exhibits apoptotic activity $(19,20)$. $\mathrm{B} 19$ has been hypothesized to be associated with rheumatic diseases including AOSD $(21,22)$. The present study aimed to investigate whether B19 serves a role as an activator of NLRP3-inflammasome in AOSD.

The present study investigated whether mRNA levels of NLRP-inflammasomes differ significantly between patients with AOSD and healthy controls, whether inflammasome inhibitors influence the expression of NLRP3-inflammasome signaling components and whether B19-associated antigens serve a role as NLRPs activators and affect the expressions of inflammasome components and downstream cytokines from peripheral blood mononuclear cells (PBMCs) of patients with AOSD.

\section{Materials and methods}

Patients and ethical approval. A total of 34 patients with active untreated AOSD (mean age \pm standard deviation, $32.7 \pm 11.1$ years; 26 women and 8 men) fulfilling the Yamaguchi criteria (14) were enrolled in Taichung Veterans General Hospital, Taiwan, between April 2013 and March 2015. Patients with infections, malignancies or other rheumatic diseases were excluded. Data were obtained on the presenting clinical features using the following definitions: i) Spiking fever was defined as an elevation of tympanic temperature above $39^{\circ} \mathrm{C}$ with daily reduction in temperature into normal range or below $39^{\circ} \mathrm{C}$; ii) typical rash was presented as salmon-red maculopapular evanescent skin lesion; iii) arthritis was defined as the presence of swelling, local warmth and tenderness over the affected joints; iv) sore throat constituted subjective symptoms without redness or exudates in pharyngeal examination and v) the presence of lymphadenopathy was defined as palpable tender lymph nodes which most commonly occurred in the cervical region. Following blood sampling for the determination of NLRPs expression, all patients with AOSD received corticosteroids, non-steroidal anti-inflammatory drugs and disease-modifying anti-rheumatic drugs. A total of 14 healthy volunteers (33.4 \pm 5.7 years of age; 12 women and 2 men), with no rheumatic diseases, were enrolled as controls. The present study was approved by the Institutional Review Board of Taichung Veterans General Hospital (Taichung, Taiwan; approval no. CF11309) and all participants submitted written informed consent according to guidelines of the ethical principles for medical research involving human subjects of the Declaration of Helsinki.

Determination of mRNA levels of NLRPs in PBMCs using reverse transcription-quantitative polymerase chain reaction (RT-qPCR). PBMCs were isolated from venous blood using
Ficoll-Paque PLUS (GE Healthcare, Chicago, IL, USA) density gradient centrifugation. Total RNA was obtained from PBMCs using the guanidinium isothiocyanate method, as previously described (23). A total of $2.5 \mu \mathrm{g}$ RNA was reverse transcribed using 200 U Moloney murine leukemia virus reverse transcriptase (cat. no. 28025013, Fermentas; Thermo Fisher Scientific, Inc., Waltham, MA, USA), which was incubated $1 \mathrm{~h}$ at $37^{\circ} \mathrm{C}$ with $0.025 \mathrm{mM}$ oligo(dT) primer and Reverse Transcriptase Buffer (18057018; Thermo Fisher Scientific, Inc.). mRNA levels of NLRP1-NLRP14 were determined by qPCR using a TaqMan Universal Master Mix (cat. no. 4440040, Applied Biosystems; Thermo Fisher Scientific, Inc.). The catalog numbers of primers and probes for each gene were as follows: NLRP1 (Hs00248187_m1), NLRP2 (Hs01546932_m1), NLRP3 (Hs00918082_m1), NLRP4 (Hs00370499_m1), NLRP5 (Hs00411266_m1), NLRP6 (Hs00373246_m1), NLRP7 (Hs00373683_m1), NLRP8 (Hs00603419_m1), NLRP9 (Hs00603423_m1), NLRP10 (Hs00738590_m1), NLRP11 (Hs00935472_m1), NLRP12 (Hs00536435_m1), NLRP13 (Hs00603406_m1), NLRP14 (Hs00698226_m1), ASC (PYCARD) (Hs00741684_g1), caspase-1 (Hs00354832_m1), IL-1ß (Hs01555410_m1), and IL-18 (Hs01038788_m1) (Applied Biosystems; Thermo Fisher Scientific, Inc.). Amplification cycles were conducted for $95^{\circ} \mathrm{C}$ for $10 \mathrm{~min}$, followed by 40 cycles of denaturation at $95^{\circ} \mathrm{C}$ for $15 \mathrm{sec}$, then annealing and extension at $60^{\circ} \mathrm{C}$ for $1 \mathrm{~min}$. To standardize mRNA levels of inflammasome components and downstream cytokines, mRNA levels of the housekeeping gene GAPDH (Hs99999905_m1) were also determined in parallel for each sample. Relative mRNA levels of NLRPs were calculated using the comparative threshold cycle $(\mathrm{Cq})$ method, $2^{-\Delta \Delta \mathrm{Cq}}(24)$, using the following equation: $\mathrm{DDCq}=$ patient $\left(\mathrm{Cq}_{\text {NLRPs gene }}-\mathrm{Cq}_{\mathrm{GAPDH}}\right)$-mean of controls $\left(\mathrm{Cq}_{\text {NLRPs gene }}-\mathrm{Cq} \mathrm{GAPDH}_{\mathrm{G}}\right)$.

Ex vivo cytokine production in PBMCs treated with inflammasome inhibitors. To verify the association between NLRP3-inflammasome and downstream inflammatory responses, alterations were determined in mRNA expression and supernatant levels of downstream cytokines in PBMCs treated with inflammasome inhibitors. PBMC samples were incubated at $37^{\circ} \mathrm{C}$ in a $5 \% \mathrm{CO}_{2}$ humidified atmosphere for $24 \mathrm{~h}$ in dimethyl sulfoxide (D2650, Sigma-Aldrich; Merck KGaA, Darmstadt, Germany) as a solvent or in the presence of inflammasome inhibitors. The inhibitors used in the present study included the NLRP3 inhibitor glibenclamide (20 $\mu \mathrm{M}$; IMI-2319, Novus Biologicals LLC, Littleton, CO, USA) and caspase-1 inhibitor VX-765 (60 $\mu \mathrm{M}$; orb61303, Biorbyt Ltd. Cambridge, $\mathrm{UK})$ at $37^{\circ} \mathrm{C}$ in a $5 \% \mathrm{CO}_{2}$ humidified atmosphere for $24 \mathrm{~h}$. The mRNA expression levels of NLRP3 were determined as described in the previous section. Cell-free supernatant was harvested by centrifugation at $4^{\circ} \mathrm{C}$ for $15 \mathrm{~min}$ at $500 \mathrm{x} \mathrm{g}$ and stored at $-70^{\circ} \mathrm{C}$ until used for the determination of cytokine levels. Levels of IL-1 $\beta$ and IL-18 were determined using ELISA kits (cat. no. ELH-IL1b-1, RayBiotech Inc., Norcross, GA, USA; and cat. no. 7620, MBL International Co., Woburn, MA, USA, respectively) according to the manufacturers' instructions.

Ex vivo assay to investigate the effect of B19-associated antigens on $M R N A$ expression levels of NLRP3 and downstream cytokines. PBMCs from 14 patients with AOSD and 4 healthy 

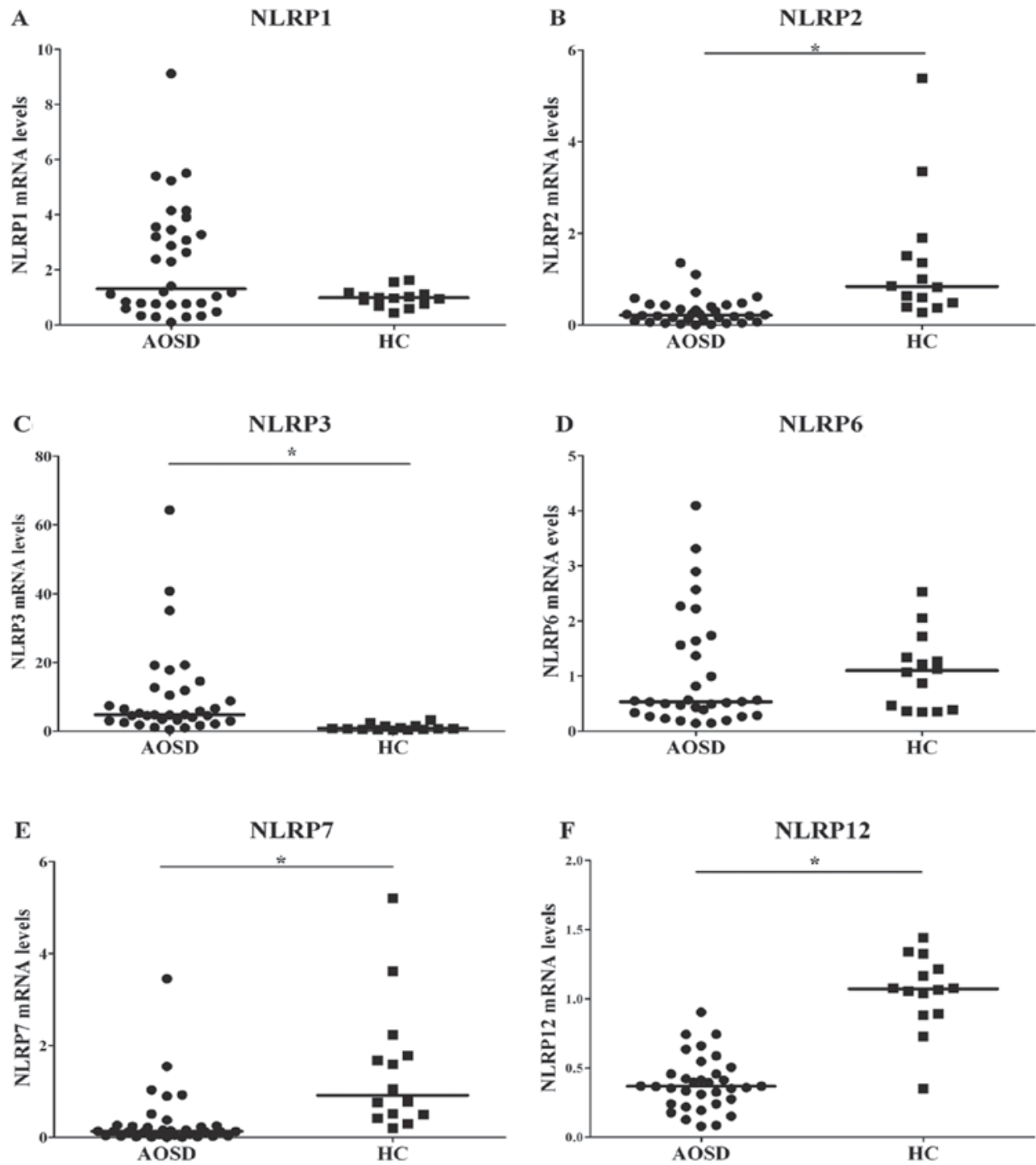

Figure 1. Comparisons of relative mRNA levels of (A) NLRP1, (B) NLRP2, (C) NLRP3, (D) NLRP6, (E) NLRP7 and (F) NLRP12 between 34 patients with AOSD and 14 HCs. Horizontal bar indicates the median value. "P<0.001. NLRP, NLR family pyrin domain containing; AOSD, adult-onset Still's disease; HC, healthy control.

volunteers were re-suspended in RPMI 1640 medium (Thermo Fisher Scientific, Inc.) supplemented with 100 units/ml penicillin, $100 \mu \mathrm{g} / \mathrm{ml}$ streptomycin and $10 \%$ fetal bovine serum (Thermo Fisher Scientific Inc.) at a final concentration $5 \times 10^{6}$ cells/well. Expression levels of NLRP3 were examined in PBMCs treated with B19-associated antigens, including $1.5 \mu \mathrm{g} / \mathrm{ml} \mathrm{B} 19-\mathrm{NS} 1$ and $1.5 \mu \mathrm{g} / \mathrm{ml} \mathrm{B} 19-\mathrm{VP} 1 \mathrm{u}$ for $24 \mathrm{~h}$, purified as previously described (25). Fold change values represent the ratio of mRNA expression levels in cells treated with B19 antigens, compared with mRNA levels in cells treated with medium only. Data are presented as the mean \pm standard deviation. mRNA levels of NLRP3 and supernatant levels of IL-1 $\beta$ and IL-18 were determined as described above.

Ex vivo assay to investigate the effect of B19-associated antigens on protein expression levels of NLRP3 and downstream cytokines. The PBMCs were treated with Protein Extraction Solution (cat. no. 17081, iNtRON Biotechnology, Seongnam, South Korea) for $20 \mathrm{~min}$ on ice and centrifuged at $10,000 \mathrm{x} \mathrm{g}$ for $30 \mathrm{~min}$ at $4^{\circ} \mathrm{C}$, proteins were quantified with a Bicinchoninic Acid Protein Assay (cat. no. 786571, Geno
Technology Inc., Louis, MO, USA). Total protein extraction $(10 \mu \mathrm{g})$ was performed from PBMCs treated with $1.5 \mu \mathrm{g} / \mathrm{ml}$ B19-NS1 and $1.5 \mu \mathrm{g} / \mathrm{ml} \mathrm{B19-VP1u}$. Proteins were separated by $10 \%$ SDS-PAGE in running buffer $(25 \mathrm{mM}$ Tris, $192 \mathrm{mM}$ glycine, $0.1 \%$ SDS). The gel was run at $70 \mathrm{~V} 30 \mathrm{~min}$ at room temperature, then at $100 \mathrm{~V}$ until the blue-dye front approached the end of the gel. The gel was transferred to polyvinylidene fluoride membranes (1620177; Bio-Rad Laboratories, Inc., Hercules, CA, USA), which were blocked with transfer buffer (50 mM Tris, $384 \mathrm{mM}$ glycine, $20 \%$ methanol) at $4^{\circ} \mathrm{C}$ for $300 \mathrm{~mA} 1 \mathrm{~h}$ with the Mini TBC Electrophoretic Transfer Cell (170-3930, Bio-Rad Laboratories, Inc.). The membranes were blocked with 5\% BSA (A7906; Sigma-Aldrich; Merck KGaA) in TBST [ $150 \mathrm{mM} \mathrm{NaCl}, 20 \mathrm{mM}$ Tris- $\mathrm{HCl}$ (pH 7.4), $0.1 \%$ Tween-20] at room temperature for $1 \mathrm{~h}$. Western blot analyses were performed using primary antibodies against NLRP3 (1:1,000; ab16097, Abcam, Cambridge, MA, USA), caspase-1 (1:1,000; NB100-56565SS, Novus Biologicals, LLC), IL-1 $\beta$ (1:1,000; NBS2-27342SS, NB100-56565SS, Novus Biologicals, LLC), IL-18 (1:1,000; PM014, MBL International Co.), and GAPDH (1:5,000; sc-32233, Santa Cruz Biotechnology, 
Inc., Dallas, TX, USA) at $4^{\circ} \mathrm{C}$ overnight. Horseradish peroxidase-conjugated anti-mouse (1:10,000; sc-2005, Santa Cruz Biotechnology, Inc.) or rabbit immunoglobulin $\mathrm{G}$ was used as a secondary antibody (1:10,000; sc-2004, Santa Cruz Biotechnology, Inc.) for $1 \mathrm{~h}$ at room temperature. Expression levels of GAPDH served as an internal control. Immunoreactive bands were detected on radiographic films using the enhanced chemiluminescence detection system (Advansta Inc., Menlo Park, CA USA). ImageJ v1.44p software (National Institutes of Health, Bethesda, MD, USA) was used for densitometry and values were normalized to GAPDH expression levels. The protein expression levels of NLRP3 and downstream cytokines were normalized to GAPDH and values are expressed relative to the control group.

Statistical analysis. Data $(\mathrm{n}=2)$ are presented as the mean \pm standard deviation or the median and interquartile range. Nonparametric Mann-Whitney U test was used for between-group comparisons of expression levels of NLRPs and downstream cytokines. Nonparametric one-way analysis of variance with Dunn-Bonferroni post-hoc tests were used for between-group comparisons of alterations in protein expression levels of NLRP3 in PBMCs stimulated with B19-VP1u, B19-NS1 or the control group. Association coefficient was calculated using the nonparametric Spearman's rank association test. Wilcoxon signed rank test was used to compare expression levels of NLRP3 and downstream cytokines prior to and following treatment. $\mathrm{P}<0.05$ was considered to indicate a statistically significant difference.

\section{Results}

Clinical characteristics of patients with AOSD. Based on the findings of physical examination, the presence of spiking fever (body temperature $\geq 39^{\circ} \mathrm{C}$ ), evanescent rash, arthritis, sore throat and lymphadenopathy were observed in 33, 30, 25, 24 and 13 of 34 patients with AOSD, respectively. Neither the age at entry nor the proportion of females differed significantly between the patients with AOSD and the healthy controls.

Comparison of mRNA levels of NLRPs between patients with $A O S D$ and controls. Significantly increased mRNA levels of NLRP3 were observed in patients with AOSD compared with healthy patients $(\mathrm{P}<0.001$; Fig. 1 and Table I). By contrast, significantly lower transcript levels of NLRP2, 7 and 12 were observed in patients with AOSD compared with the healthy patients. Transcript levels of NLRP1 and 6 did not differ significantly in patients with AOSD compared with the control group. The mRNA levels of NLRP4, 5, 8, 9, 10, 11, 13 and 14 were too low to be detected by qPCR in patients with AOSD or healthy controls.

Association between NLRP3 mRNA expression levels and clinical features in AOSD. Higher levels of NLRP3 expression were observed in AOSD patients with skin rash or arthritis compared with in those without $(10.91 \pm 13.84$ vs. $3.66 \pm 2.09 ; 12.11 \pm 14.82$ vs. 4.37 \pm 3.20 ; respectively); statistical significance was not observed $(\mathrm{P}=0.309, \mathrm{P}=0.134$, respectively). In addition, no significant differences in NLRP3 expression levels between
Table I. Relative mRNA levels of NLRPs in patients with AOSD and healthy controls.

\begin{tabular}{lc}
\hline NLRP & $\begin{array}{c}\text { Relative mRNA expression } \\
\text { levels [median (IQR) fold] }\end{array}$ \\
\hline NLRP1 & $1.31(0.76-3.48)$ \\
AOSD & $0.99(0.74-1.14)$ \\
Control & \\
NLRP2 & $0.22(0.08-0.44)^{\mathrm{a}}$ \\
AOSD & $0.84(0.47-1.61)$ \\
Control & \\
NLRP3 & $4.79(3.04-2.05)^{\mathrm{a}}$ \\
AOSD & $0.79(0.61-1.58)$ \\
Control & \\
NLRP6 & $0.53(0.30-1.62)$ \\
AOSD & $1.10(0.38-1.44)$ \\
Control & \\
NLRP7 & $0.13(0.04-0.25)^{\mathrm{a}}$ \\
AOSD & $0.91(0.48-1.89)$ \\
Control & \\
NLRP12 & $0.37(0.24-0.47)^{\mathrm{a}}$ \\
AOSD & $1.07(0.89-1.24)$ \\
Control & \\
\hline
\end{tabular}

${ }^{\mathrm{a}} \mathrm{P}<0.001$, vs. control group. AOSD, adult-onset Still's disease; IQR, interquartile range; NLRP, NLR family pyrin domain containing.

AOSD patients with and without sore throats were observed $(11.39 \pm 15.10$ vs. $6.87 \pm 6.38, \mathrm{P}=0.372)$, or between AOSD patients with and without lymphadenopathy $(11.59 \pm 12.68$ vs. $9.11 \pm 13.73, \mathrm{P}=0.601)$.

Correlation between $m R N A$ levels of NLRPs and expression of downstream inflammasome signaling components in patients with AOSD. NLRP3 transcript levels were positively associated with expression levels of inflammasome signaling components and downstream cytokines including PYCARD (correlation coefficient $r=0.343, \mathrm{P}<0.05)$, caspase $-1(\mathrm{r}=0.445, \mathrm{P}<0.01)$, IL-1 $\beta$ $(\mathrm{r}=0.379, \mathrm{P}<0.05)$ and IL-18 $(\mathrm{r}=0.482, \mathrm{P}<0.01)$ in patients with AOSD. Conversely, NLRP2 transcript levels were inversely correlated with expression levels of PYCARD ( $r=-0.389$, $\mathrm{P}<0.05)$, caspase-1 ( $\mathrm{r}=-0.515, \mathrm{P}<0.01)$, IL-1 $\beta(\mathrm{r}=-0.351, \mathrm{P}<0.05)$ and IL-18 $(\mathrm{r}=-0.481, \mathrm{P}<0.01)$ in patients with AOSD.

NLRP3 and caspase-1 are associated with inflammasome signaling in AOSD. Inflammatory response to inflammasome inhibitors was evaluated to analyze the NLRP3-inflammasome signaling pathway. The results obtained indicated that treatment with NLRP3 inhibitor significantly downregulated mRNA levels of NLRP3, caspase-1, IL-1 $\beta$ and IL-18 (all P<0.005) in PBMCs from patients with AOSD (Fig. 2A). Similarly, treatment with caspase-1 inhibitor significantly reduced mRNA levels of caspase-1, IL-1 $\beta$ and IL-18 (all $\mathrm{P}<0.005$; Fig. 2B). Treatment of PBMCs from patients with AOSD with NLRP3 inhibitor significantly reduced IL-1 $\beta$ and IL-18 

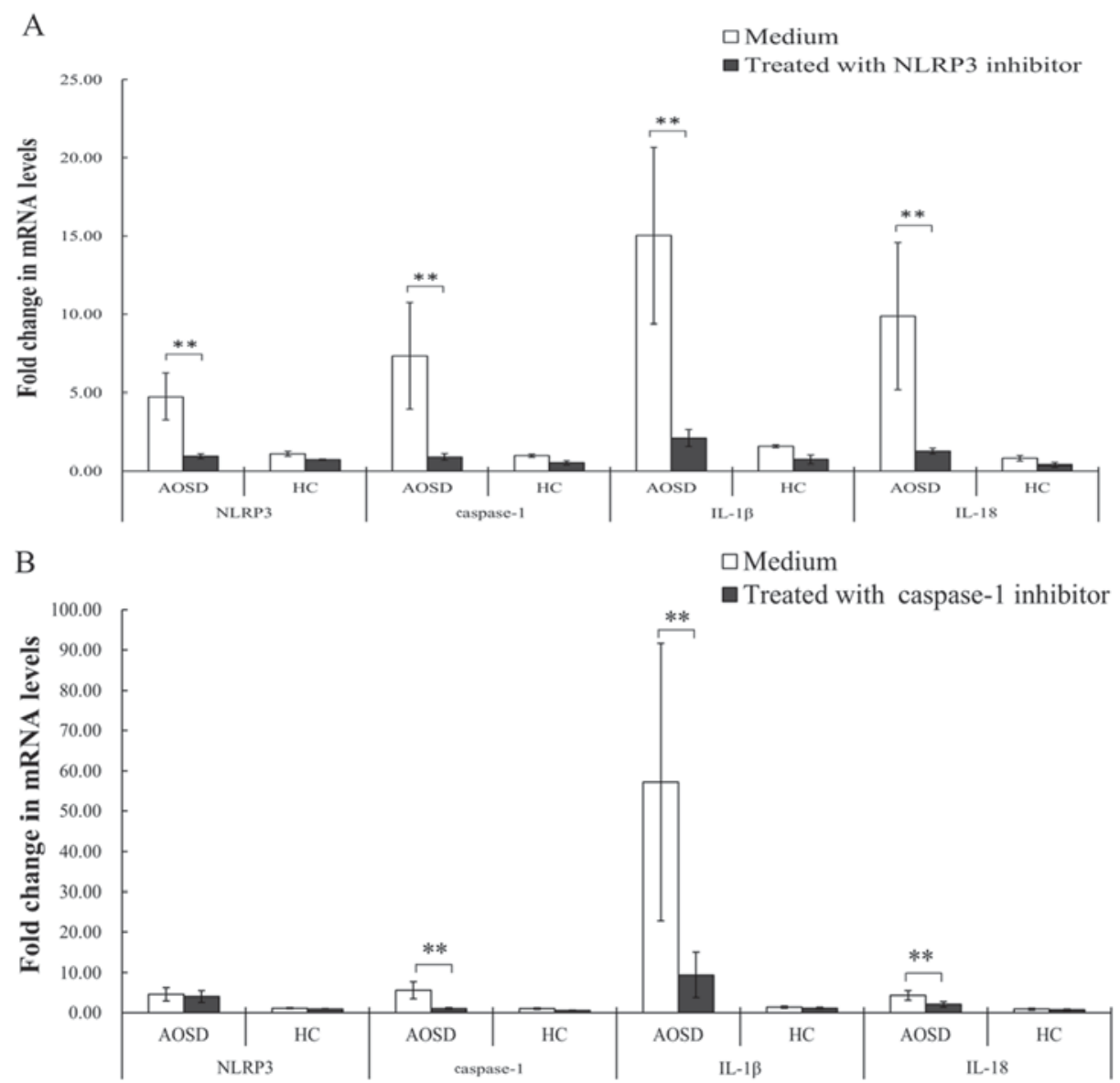

$\mathrm{C}$

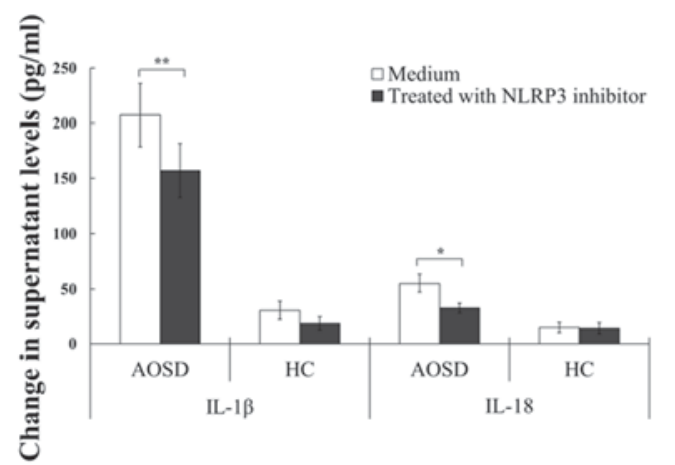

$\mathrm{D}$

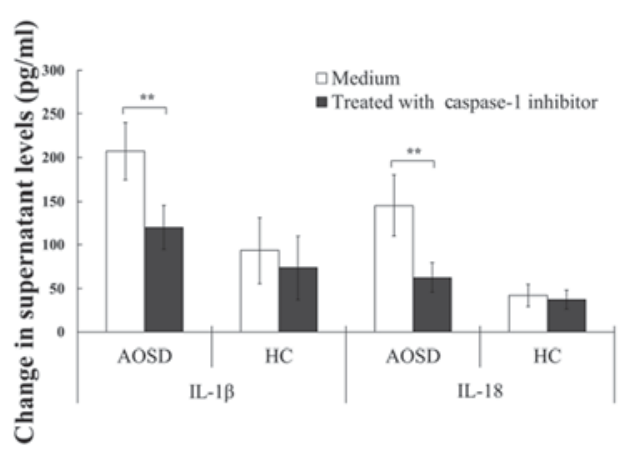

Figure 2. Fold changes in mRNA levels of NLRP3, caspase-1, IL-1 $\beta$ and IL-18 in PBMCs treated with (A) NLRP3 inhibitor glibenclamide and (B) caspase-1 inhibitor VX-765 in patients with AOSD and HCs. Alterations in supernatant levels of IL-1 $\beta$ and -18 in PBMCs culture treated with (C) NLRP3 inhibitor glibenclamide and (D) caspase-1 inhibitor VX-765 in patients with AOSD and HC. Data are presented as the mean \pm standard deviation. "P $<0.05$, "* P $<0.005$. AOSD, adult-onset Still's disease; HC, healthy control; IL, interleukin; NLRP, NLR family pyrin domain containing.

levels $(\mathrm{P}<0.005$ and $\mathrm{P}<0.05$, respectively) in the supernatant, compared with the control treatment group (Fig. 2C). Similarly, treatment of PBMCs from patients with AOSD with caspase-1 inhibitor significantly reduced levels of IL-1 $\beta$ and -18, compared with the control group (both $\mathrm{P}<0.005$; Fig. 2D).

Expression levels of NLRP3-inflammasome signaling components in PBMCs are stimulated by parvovirus $B 19$ antigens. To investigate the effect of B19 antigens on NLRP3 activation, PBMC cells were treated with B19 antigens and fold changes relative to control in transcript levels of NLRP3 and supernatant levels of downstream cytokines were determined. The results of the present study revealed that stimulation of PBMCs from patients with AOSD with B19-NS1 induced significant increases in NLRP3, caspase-1 and IL-1 $\beta$ compared with healthy patients (all $\mathrm{P}<0.0005$; Fig. 3A). However, transcript levels of NLRP3 and caspase-1 in B19-VP1u-stimulated PBMCs did not differ significantly between patients with AOSD and healthy controls. B19-NS1 treatment significantly increased supernatant levels of IL-1 $\beta$ in patients with AOSD compared with healthy controls $(\mathrm{P}<0.05)$. The difference in IL-18 levels was not statistically different (Fig. 3B). 

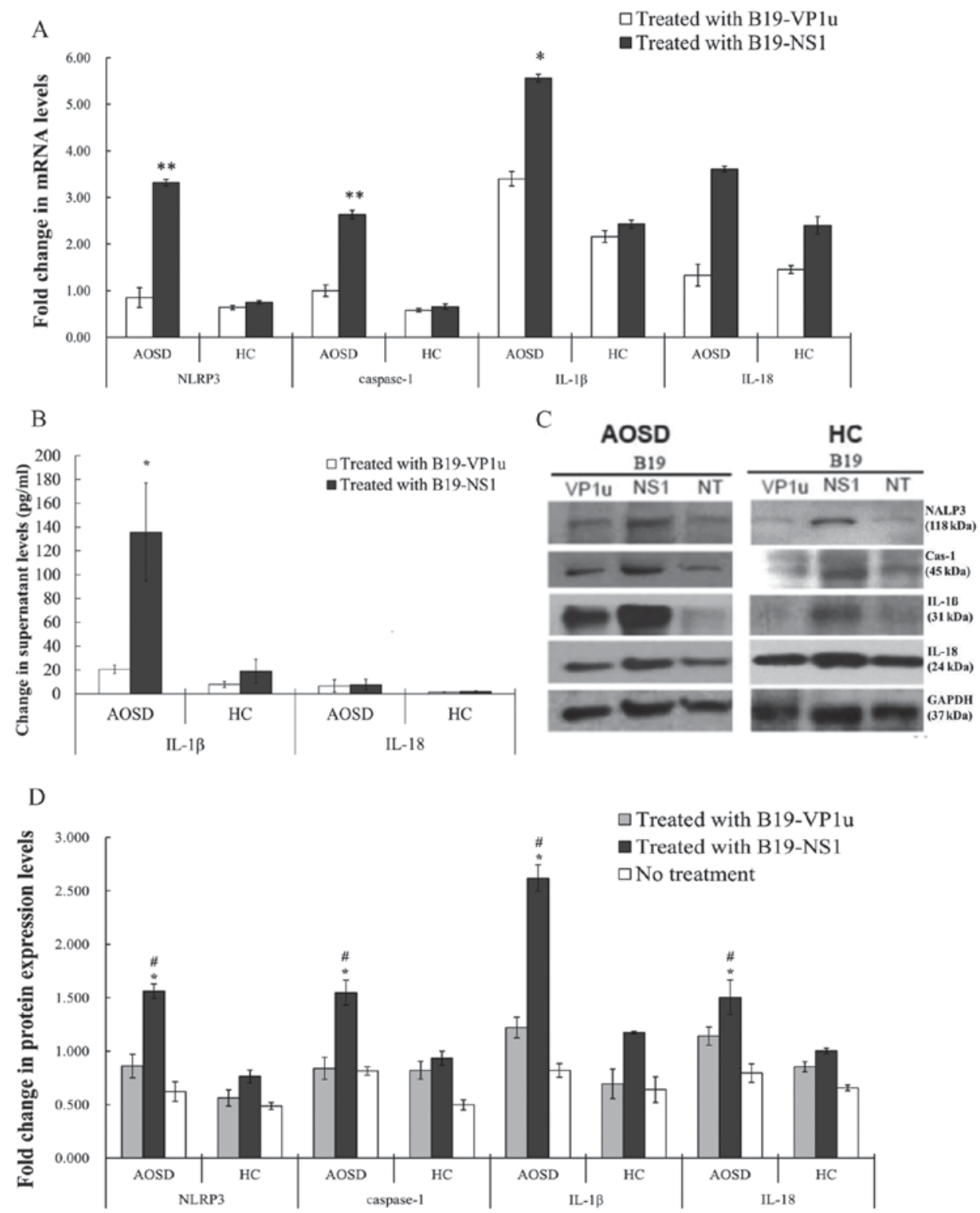

Figure 3. Alterations in mRNA and protein expression levels following treatment with B19-VP1u and B19-NS1. (A) Fold-changes, relative to the healthy control group, in transcript levels of NLRP3, caspase-1, IL-1 $\beta$ and-18 in PBMCs stimulated with B19-VP1u and B19-NS1 in patients with AOSD and HCs. (B) Supernatant levels of IL-1 $\beta$ and IL-18 in PBMCs stimulated with B19-VP1u and B19-NS1 in patients with AOSD and HCs. (C) Western blot analysis of expression of NLRP3, Cas-1, IL-1 $\beta$ and-18 and control GAPDG proteins in PBMC lysates obtained from one AOSD patient and one HC. (D) Protein expression levels (fold-changes relative to healthy control group) of NLRP3, caspase-1, IL-1 $\beta$ and-18 in PBMCs stimulated with B19-VP1u and B19-NS1 in patients with AOSD and HC. Data are presented as the mean \pm standard deviation. ${ }^{*} \mathrm{P}<0.05$ and ${ }^{* *} \mathrm{P}<0.005$ vs. the respective $\mathrm{HC}$ group, ${ }^{\#} \mathrm{P}<0.05$, vs. the respective no treatment group. PBMC, peripheral blood mononuclear cell; AOSD, adult-onset Still's disease; HC, healthy control; IL, interleukin; NLRP3, NLR family pyrin domain containing 3; Cas-1, caspase-1; B19-VP1u, human parvovirus B19 VP1-unique region; B19-NS1, human parvovirus B19 non-structural protein-1.

Patients with AOSD exhibit increased protein expression of NLRP3-inflammasome components in PBMC lysates stimulated with B19-NS1, compared with samples treated with B19-VP1u and medium only. Stimulation of PBMCs with B19-NS1 induced an increase in the expression of NLRP3, caspase-1, IL-1 $\beta$ and IL-18 in patients with AOSD compared with healthy controls (all $\mathrm{P}<0.05$; Fig. $3 \mathrm{C}$ and $\mathrm{D}$ ).

\section{Discussion}

Different types of inflammasomes contain different NLRPs responsible for variable regulation of inflammatory responses $(2,4,26)$. The present study demonstrated markedly increased NLRP3 transcript levels in patients with AOSD compared with healthy controls. NLRP3 expression levels were positively associated with levels of inflammasome components and downstream cytokines in patients with AOSD. As previously demonstrated, the results of the present study confirmed that activation of NLRP3 and caspase-1 is essential for the inflammatory response in AOSD (27). The above observations suggest involvement of NLRP3-inflammasome signaling in AOSD pathogenesis. Parvovirus B19 infection has been previously associated with AOSD and B19-NS1 has been hypothesized to serve role in NLRP3 activation in patients with AOSD (22).

Following activation of NLRP3 and caspase-1, release of IL-1 $\beta$ and IL-18 is the final stage of pathogenesis 
of inflammasome-associated AIDs. NLRP3 transcript levels were positively correlated with the expression levels of inflammasome signaling components in patients with AOSD. Furthermore, NLRP3 and caspase-1 inhibitors markedly suppressed expression of IL- $1 \beta$ and IL-18, indicating that NLRP3-associated caspase-1-dependent mechanism may mediate the production of downstream cytokines in AOSD.

mRNA levels of NLRP2, a member of negative regulatory NLRPs, were inversely correlated with the expression levels of downstream inflammasome signaling components in patients with AOSD. In addition, a negative correlation was identified between NLRP2 levels and NLRP3 levels in patients with AOSD (data not presented), supporting a hypothesis that NLRP2 competes with NLRP3 in binding to PYCARD and subsequently activating caspase-1 $(28,29)$. The aforementioned observations suggest that NLRP2 may serve a role as a negative regulator of immune responses in AOSD. However, the role of NLRP2-inflammasome in rheumatic patients remains to be elucidated.

mRNA levels of NLRP7 and NLRP12 were lower in patients with AOSD compared with healthy controls. Although it remains to be elucidated whether NLRP7 is an activator or an inhibitor of inflammasome, it has been hypothesized that NLRP7 can inhibit NLRP3 and caspase-1-mediated release of IL-1 $\beta$ (30-32). NLRP12 has been previously reported to suppress the production of proinflammatory cytokines by inhibiting nuclear factor- $\mathrm{\kappa B}$ via a proteasome-dependent pathway and has been identified as a negative regulator of inflammation (33).

Although in a previous report NLRP9 has been implicated in the pathogenesis of AOSD and NLRP10 has been previously identified as an inflammasome inhibitor $(34,35)$, mRNA levels of NLRP4, 5, 8, 9, 10,11, 13, and 14 were undetectable in the present study. This observation supports the hypothesis that only a few mammalian NLRPs directly participate in inflammatory responses and certain NLRPs may be involved in other non-immune pathways including embryogenesis (36). Another possibility is that the assay used in the present study demonstrated limited sensitivity and therefore failed to detect certain NLRPs.

Certain pathogen associated molecular patterns have been identified as NLRP3 activators $(1,3)$ but specific ligands of NLRP3 in AOSD remain to be elucidated. Based on the association of parvovirus B19 infection with AOSD pathogenesis, we hypothesized that B19-associated antigens may activate the expression of NLRP3-inflammasome associated signaling (22). Ex vivo experiments included in the present study are, to the best of our knowledge, the first to reveal that B19-NS1 can up-regulate the expression of mRNA and protein levels of NLRP3, indicating that B19-NS1 may be an activator of NLRP3 in AOSD. The results of the present study confirm previous hypotheses that adenoviruses (non-enveloped DNA viruses) can activate NLRP3 and that NLRP3 may be implicated in immune responses to viral infection $(1,37)$.

There were certain limitations of the present study. Due to difficulties associated with obtaining biopsy tissues, expression of NLRPs was not investigated in lesion specimens from patients with AOSD. Lack of association between expression levels of NLRP3 and clinical features may be due to the small sample size in this clinically heterogeneous and uncommon disease. Association between NLRP3 levels and parvovirus B19 DNA was not investigated in the present study.

Enhanced expression of NLRP3 resulting in elevated levels of downstream cytokines may be involved in pathogenesis of AOSD. Since activators of NLRP3 in AOSD remain to be elucidated, the presented study provides novel insights into the innate immunity of AOSD.

\section{Acknowledgements}

The present study was supported by the National Science Council of Taiwan (grant nos. NSC-101-2314-B-010-030-MY3 and NSC-101-2314-B-040-008) and by the Taichung Veterans General Hospital and National Yang-Ming University (grant. no. TCVGH-YM1010204). The authors of the present study would also like to thank Mr. Ted Knoy (Writing Center, Hsinchu, Taiwan, R.O.C.) for his editorial assistance.

\section{References}

1. Muruve DA, Pétrilli V, Zaiss AK, White LR, Clark SA, Ross PJ, Parks RJ and Tschopp J: The inflammasome recognizes cytosolic microbial and host DNA and triggers an innate immune response. Nature 452: 103-107, 2008.

2. Martinon F, Gaide O, Pétrilli V, Mayor A and Tschopp J: NALP inflammasome: A central role in innate immunity. Semin Immunopathol 29: 213-229, 2007.

3. Schroder K and Tschopp J: The inflammasomes. Cell 140: 821-832, 2010.

4. Tschopp J, Martinon F and Burns K: NALPs: A novel protein family involved in inflammation. Nat Rev Mol Cell Biol 4: 95-104, 2003

5. Ting JP, Lovering RC, Alnemri ES, Bertin J, Boss JM, Davis BK, Flavell RA, Girardin SE, Godzik A, Harton JA, et al: The NLR gene family: A standard nomenclature. Immunity 28: 285-287, 2008.

6. Sidiropoulos PI, Goulielmos G, Voloudakis GK, Petraki E and Boumpas DT: Inflammasomes and rheumatic diseases: Evolving concepts. Ann Rheum Dis 67: 1382-1389, 2008.

7. Busso $\mathrm{N}$ and So A: Microcrystals as DAMPs and their role in joint inflammation. Rheumatology (Oxford) 51: 1154-1160, 2012.

8. Varghese GP, Uporova L, Halfvarson J, Sirsjö A and Fransén K: Polymorphism in the NLRP3 inflammasome-associated EIF2K2 gene and inflammatory bowel disease. Mol Med Rep 11: 4579-4584, 2015

9. McDermott MF, Aksentijevich I, Galon J, McDermott EM, Ogunkolade BW, Centola M, Mansfield E, Gadina M, Karenko L, Pettersson T, et al: Germline mutations in the extracellular domains of the $55 \mathrm{kDa}$ TNF receptor, TNFR1, define a family of dominantly inherited autoinflammatory syndromes. Cell 97: 133-144, 1999.

10. Masters SL, Simon A, Aksentijevich I and Kastner DL: Horror autoinflammaticus: The molecular pathophysiology of autoinflammatory disease ("). Annu Rev Immunol 27: 621-668, 2009.

11. Kastner DL, Aksentijevich I and Goldbach-Mansky R: Autoinflammatory disease reloaded: A clinical perspective. Cell 140: 784-790, 2010.

12. Gerfaud-Valentin M, Jamilloux Y, Iwaz J and Sève P: Adult-onset Still's disease. Autoimmun Rev 13: 708-722, 2014.

13. Kadavath $S$ and Efthimiou P: Adult-onset Still's disease-pathogenesis, clinical manifestations, and new treatment options. Ann Med 47: 6-14, 2015.

14. Yamaguchi M, Ohta A, Tsunematsu T, Kasukawa R, Mizushima Y, Kashiwagi H, Kashiwazaki S, Tanimoto K, Matsumoto Y, Ota T, et al: Preliminary criteria for classification of adult Still's disease. J Rheumatol 19: 424-430, 1992.

15. Kawashima M, Yamamura M, Taniai M, Yamauchi $H$, Tanimoto T, Kurimoto M, Miyawaki S, Amano T, Takeuchi T and Makino $\mathrm{H}$ : Levels of interleukin-18 and its binding inhibitors in the blood circulation of patients with adult-onset Still's disease. Arthritis Rheum 44: 550-560, 2001. 
16. Choi JH, Suh CH, Lee YM, Suh YJ, Lee SK, Kim SS, Nahm DH and Park HS: Serum cytokine profiles in patients with adult onset Still's disease. J Rheumatol 30: 2422-2427, 2003.

17. Chen DY, Chen YM, Lan JL, Lin CC, Chen HH and Hsieh CW: Potential role of Th17 cells in the pathogenesis of adult-onset still's disease. Rheumatology (Oxford) 49: 2305-2312, 2010.

18. Priori R, Barone F, Alessandri C, Colafrancesco S, McInnes IB, Pitzalis C, Valesini G and Bombardieri M: Markely increased IL-18 liver expression in adult-onset Still's disease-related hepatitis. Rheumatology (Oxford) 50: 776-780, 2011.

19. Young NS and Brown KE: Parvovirus B19. N Engl J Med 350: $586-597,2004$

20. Dorsch S, Liebisch G, Kaufmann B, von Landenberg P, Hoffmann JH, Drobnik W and Modrow S: The VP1 unique region of parvovirus $\mathrm{B} 19$ and its constituent phospholipase A2-like activity. J Virol 76: 2014-2018, 2002.

21. Adamson-Small LA, Ignatovich IV, Laemmerhirt MG and Hobbs JA: Persistent parvovirus B19 infection in non-erythroid tissues: Possible role in the inflammatory and disease process. Virus Res 190: 8-16, 2014.

22. Chen DY, Chen YM, Lan JL, Tzang BS, Lin CC and Hsu TC: Significant association of past parvovirus B19 infection with cytopenia in both adult-onset Still's disease and systemic lupus erythematosus patients. Clin Chim Acta 413: 855-860, 2012

23. Chomczynski P and Sacchi N: Single-step method of RNA isolation by acid guanidinium thiocyanate-phenol-chloroform extraction. Anal Biochem 162: 156-159, 1987.

24. Livak KJ and Schmittgen TD: Analysis of relative gene expression data using real-time quantitative PCR and the 2(-Delta Delta C(T)) method. Methods 25: 402-408, 2001

25. Tzang BS, Tsay GJ, Lee YJ, Li C, Sun YS and Hsu TC: The association of VP1 unique region protein in acute parvovirus B19 infection and anti-phospholipid antibody production. Clin Chim Acta 378: 59-65, 2007.

26. Pontillo A, Girardelli M, Kamada AJ, Pancotto JA, Donadi EA, Crovella S and Sandrin-Garcia P: Polymorphisms in inflammasome genes are involved in the predisposition to systemic lupus erythematosus. Autoimmunity 45: 271-278, 2012.

27. Tschopp J and Schroder K: NLRP3 inflammasome activation: The convergence of multiple signaling pathways on ROS production. Nat Rev Immunol 10: 210-215, 2010.
28. Bostanci N, Emingil G, Saygan B, Turkoglu O, Atilla G, Curtis MA and Belibasakis GN: Expression and regulation of the NALP3 inflammasome complex in periodontal diseases. Clin Exp Immunol 157: 415-422, 2009.

29. Bruey JM, Bruey-Sedano N, Newman R, Chandler S, Stehlik C and Reed JC: PAN1/NALP2/PYPAF2, an inducible inflammatory mediator that regulates NF-kappaB and caspase-1 activation in macrophages. J Biol Chem 279: 51897-51907, 2004.

30. Radian AD, de Almeida L, Dorfleutner A and Stehlik C: NLRP7 and related inflammasome activating pattern recognition receptors and their function in host defense and disease. Microbes Infect 15: 630-639, 2013.

31. Khare S, Dorfleutner A, Bryan NB, Yun C, Radian AD, de Almeida L, Rojanasakul Y and Stehlik C: An NLRP7-containing inflammasome mediates recognition of microbial lipopeptides in human macrophages. Immunity 36 : 464-476, 2012.

32. Kinoshita T, Wang Y, Hasegawa M, Imamura R and Suda T: PYPAF3, a PYRIN-containing APAF-1-like protein, is a feedback regulator of caspase-1-dependent interleukin-1beta secretion. J Biol Chem 280: 21720-21725, 2005.

33. Pinheiro AS, Eibl C, Ekman-Vural Z, Schwarzenbacher R and Peti W: The NLRP12 pyrin domain: Structure, dynamics, and functional insights. J Mol Biol 413: 790-803, 2011.

34. Tadaki H, Saitsu H, Nishimura-Tadaki A, Imagawa T, Kikuchi M, Hara R, Kaneko U, Kishi T, Miyamae T, Miyake N, et al: De novo 19q13.42 duplications involving NLRP gene cluster in a patient with systemic-onset juvenile idiopathic arthritis. J Hum Genet 56: 343-347, 2011.

35. Wang Y, Hasegawa M, Imamura R, Kinoshita T, Kondo C, Konaka K and Suda T: PYNOD, a novel Apaf-1/CED4-like protein is an inhibitor of ASC and caspase-1. Int Immunol 16: 777-786, 2004

36. Fernandes R, Tsuda C, Perumalsamy AL, Naranian T, Chong J, Acton BM, Tong ZB, Nelson LM and Jurisicova A: NLRP5 mediates mitochondrial function in mouse oocytes and embryos. Biol Reprod 86: 138, 1-10, 2012.

37. Barlan AU, Griffin TM, McGuire KA and Wiethoff CM: Adenovirus membrane penetration activates the NLRP3 inflammasome. J Virol 85: 146-155, 2011. 